\title{
ARTICLE Disconnection of drug-response and placebo-response in acute-phase antipsychotic drug trials on schizophrenia? Meta-regression analysis
}

\author{
Stefan Leucht ${ }^{1}{ }^{1}$, Anna Chaimani ${ }^{2}$, Dimitris Mavridis ${ }^{3,4}$, Claudia Leucht ${ }^{1}$, Maximilian Huhn ${ }^{1}$, Bartosz Helfer (D) $^{5}$, Myrto Samara ${ }^{1}$, \\ Andrea Cipriani ${ }^{6,7}$, John R. Geddes ${ }^{6,7}$ and John M. Davis ${ }^{8,9}$
}

Differences in efficacy between antipsychotics and placebo in schizophrenia trials have decreased over the past decades. Previous studies have tried to identify potential explanatory factors focusing on response to placebo; however, it is still not clear which factors, if any, specifically moderate drug-response, as they may be different from those moderating placebo-response. Therefore, in this meta-regression analysis we explore whether there is an interaction between drug-response and placebo-response in terms of effect size. We systematically searched multiple electronic databases, ClinicalTrials.gov, and the US Food and Drug Administration website for randomized, placebo-controlled trials investigating the efficacy of antipsychotics in patients with acute schizophrenia (last update: October 2016). The main outcome was the change on the Brief Psychiatric Rating Scale or the Positive and Negative Syndrome Scale score from baseline to endpoint after at least 3 weeks of treatment. Multiple patient-, design-, and drug-related potential predictors of response were analyzed by meta-regressions, as predefined in the study protocol. Overall, 167 trials with 28,102 participants were included. Publication year, the number of participants and sites, mean dose, minimum severity threshold as an inclusion criterion, chronicity, industry sponsorship, type of rating scale, diagnostic criteria, and number of medications had a different impact on drug and placebo response. By contrast, baseline severity, duration of wash-out, study duration, and study region affected drug and placebo response in a similar way without a net effect on effect sizes. No other factors had a significant effect on either drug-response or placebo-response. In conclusion, as individual moderators may have different impact on placeboresponse and drug-response, it is important to consider also the specific factors influencing drug-response in order to fully understand the difference between antipsychotics and placebo. These results shed further light on the phenomenon of decreasing effect size of antipsychotics for schizophrenia over time and should help design future randomized controlled trials in the field (Prospero registration number CRD42013003342).

Neuropsychopharmacology (2019) 44:1955-1966; https://doi.org/10.1038/s41386-019-0440-6

\section{INTRODUCTION}

Differences in efficacy between antipsychotic drugs and placebo in clinical trials of acute schizophrenia have decreased over the decades and we have recently demonstrated that this is to large degree due to an increasing placebo response [1]. This phenomenon might help to account for some unexpected trial results where even standard drugs such as haloperidol did not outperform placebo [2]. Implications of these results pose a challenge for antipsychotic drug development, as well as for assessment of their efficacy in everyday clinical practice.

We previously have demonstrated that drug-placebo differences can be explained mainly by the increasing placebo response and to a lesser extent industry sponsorship. When we investigated factors explaining placebo-response in a subsequent paper, lower chronicity and higher sample size were correlated with this phenomenon [3], replicating some of the findings of previous publications, which were based on $\sim 2$ times smaller datasets [4-6].

However, as effect sizes are defined as a difference between drug effects and placebo effects, the remaining question is which factors moderate drug-response and whether these are different from those moderating placebo-response. Intuitively, one would assume that a factor leading to an increase in placebo response would lead to a parallel increase of drug response (this is sometimes referred to as the "additivity assumption" [6, 7]). In our original paper on drug-placebo differences, we found that the additivity assumption is not always fulfilled. While placeboresponse increased over the years, drug-response remained stable, leading to decreasing effect sizes over the decades [1].

\footnotetext{
${ }^{1}$ Department of Psychiatry and Psychotherapy, Technische Universität München, Klinikum rechts der Isar, Ismaninger Straße 22,81675 Munich, Germany; ${ }^{2}$ INSERM, UMR1153 Epidemiology and Statistics, Sorbonne Paris Cité Research Center (CRESS), METHODS Team, Paris Descartes University, Cochrane France, Paris, France; ${ }^{3}$ Department of Primary Education, University of loannina School of Education, University Campus loannina, 45110 loannina, Greece; ${ }^{4}$ Sorbonne Paris Cité, Faculté de Médecine, Paris Descartes University, Paris, France; ${ }^{5}$ Department of Medicine, Imperial College London, London W2 1PG, UK; ${ }^{6}$ Department of Psychiatry, Warneford Hospital, University of Oxford, Oxford OX3 7JX, UK;

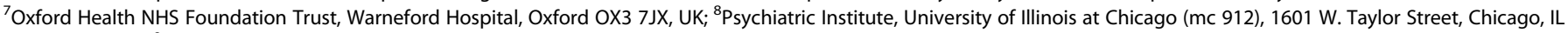
60612, USA and ${ }^{9}$ Maryland Psychiatric Research Center, Baltimore, MD, USA

Correspondence: Stefan Leucht (Stefan.Leucht@tum.de)

These authors have contributed equally: Stefan Leucht, Anna Chaimani
}

Received: 30 January 2019 Revised: 26 April 2019 Accepted: 10 June 2019

Published online: 18 June 2019 
Therefore, here we try to identify modifiers of drug-response and explore how they interplay with modifiers of placebo response in the forming of effect sizes. Our results will provide further in-depth clarification for the decrease in drug-placebo differences over the years and may have an impact on the design of future placebo-controlled clinical trials of antipsychotic drugs.

\section{MATERIAL AND METHODS}

We followed the PRISMA guidelines [8] (see checklist in the data supplement S1) and initially published a protocol in PROSPERO (CRD42013003342, see data supplement S2). We used the same database and largely the same text of the method section as in our previous publications for consistency $[1,3]$.

\section{Inclusion/exclusion criteria}

Participants. Adults with acute exacerbations of schizophrenia or related disorders (following the Cochrane Schizophrenia Group) were included. We accepted all diagnostic criteria and we also included schizoaffective, schizophreniform, or delusional disorder, because these do not require generally different treatment [9]. We excluded relapse prevention studies in stable patients receiving maintenance medication [10], studies in patients with predominant negative symptoms, and studies in patients with major concomitant somatic or psychiatric illness. There is no indication that effect sizes have decreased in relapse prevention studies in stable patients, differences between drug and placebo remain large [10]. The outcome in patients with predominant negative symptoms would be different (negative symptoms rather than overall symptoms). Studies in patients with major concomitant or psychiatric illness are extremely rare and would have increased heterogeneity even further.

Interventions. We included all antipsychotics licensed in at least one country, except clozapine, a more efficacious drug [11] so that pooling with the other compounds would not have been appropriate (only one clozapine arm with nine patients had to be excluded on this basis [12] making the impact of this decision negligible). We excluded intramuscular formulations, because these are used primarily as sources either for emergency use (short-acting i.m. drugs) or for relapse prevention (long-acting depot drugs). We examined all antipsychotics as a group under the assumption that efficacy differences between drugs are small $[11,13-15]$, except for clozapine, which was excluded for this reason.

Types of studies. Published and unpublished, double-blind, placebo-controlled randomized controlled trials of at least 3 weeks duration [16] were included. Studies with a high risk of bias in sequence generation or allocation concealment were excluded [17]. We a priori excluded Chinese studies due to quality concerns $[18,19]$.

\section{Search strategy}

We searched the Cochrane-Schizophrenia-Group-ControlledTrials-Register (compiled by regular systematic searches of more than 15 databases, clinical trial registers, the FDA website, hand searches and conference proceedings [20] without language restrictions, available to us until version August 2009) with the term "placebo;" and we searched MEDLINE, EMBASE, Psychlnfo, Cochrane CENTRAL and ClinicalTrials.gov (last search October 2016, search terms are presented in the online supplement S3), supplemented by screening previous reviews [5, 11, 21-29].

\section{Outcomes}

The outcome was the mean change from baseline to endpoint of the Positive and Negative Syndrome Scale (PANSS [30]) total score. If the PANSS was not available we used the change from baseline to endpoint of the Brief Psychiatric Rating Scale (BPRS [31]) and converted it to the PANSS using a validated method [32].

\section{Study selection and data extraction}

At least two reviewers among $\mathrm{MH}, \mathrm{MT}, \mathrm{MS}$, and SL independently selected potentially relevant publications from the abstracts found by our search and decided to include studies, and at least two reviewers among $\mathrm{CL}, \mathrm{MH}, \mathrm{BH}, \mathrm{MS}, \mathrm{MR}, \mathrm{SB}, \mathrm{MK}$, $\mathrm{PR}, \mathrm{TA}, \mathrm{NP}$, and SL (see acknowledgement) extracted data in duplicate in Excel sheets. Risk of bias was independently assessed by at least two of the following reviewers $(C L, S L$, $\mathrm{MH}, \mathrm{BH}$ ) with the Cochrane Collaboration's risk-of-bias tool [17]. Disagreement was resolved by discussion. Missing data were requested from authors or the sponsoring pharmaceutical companies for all studies published in the last 30 years. We preferably extracted intention-to-treat data and we preferred mixed-effect-model-of-repeated-measurements (MMRM) models over last-observation-carried-forward (LOCF). It is nowadays a standard that analyses should be based on intention-to-treat datasets, and it has been shown that MMRM is a more appropriate statistical approach to account for attrition in psychopharmacological trials than LOCF [33]. Missing standard deviations were estimated from test statistics or by using the mean standard deviation of the remaining studies [34, 35].

\section{Statistical analysis}

We conducted meta-regressions in a frequentist framework with drug-response as dependent variables and compared them with our previously published results on placebo-response [3] and drug-placebo differences [1]. All the potential predictors that we had used in our previous papers [1, 3] were analyzed as independent variables. Drug and placebo-response were continuous variables defined as the difference in PANSS/BPRS scale before and after treatment. Drug-placebo differences were calculated as standardized mean differences. The initial choice of predictors had been based on previous evidence [4-6, 36, 37], which suggested that these factors might be relevant. We categorized the moderators into patient-, study design-, and drug-related factors, although there were expected overlaps. We first ran univariable meta-regressions exploring separately the effect of each potential moderator. For the multivariable metaregression models we only used factors that were significant in univariable analyses and we followed a formal variable selection procedure using the backward-stepwise algorithm with removal criterion $p=0.15$. We monitored how much heterogeneity in drug response, placebo response and drug placebo differences each predictor explains by comparing the heterogeneity of each metaregression model with the heterogeneity of the model without any covariates.

Patient-related factors. The patient-related factors were: chronicity measured by the patients' mean age, duration of illness, duration of the current episode, and first-episode status [5, 37], percentage men [37], US American populations vs. not $/$ mixed countries [38], severity (PANSS total score) at baseline [36], in-vs. outpatient [5], operationalized criteria (e.g., ICD-10 or DSM-III to IVR) vs. unspecific 'clinical diagnoses", and the total dropout rates of both groups combined (newly added as a moderator) because the joint process of response with dropout is rarely accounted for appropriately $[39,40]$.

Study design-related factors. We analyzed publication year, the impact of risk of bias (appropriate vs. unclear randomization [41] and allocation concealment methods [42], blinding [42], and missing outcome data [17, 43]), study duration [5], duration of wash-out [5], requirement of a scale-derived minimum of symptoms at baseline [36], PANSS vs. BPRS as a scale, sample size [44], number of sites [5], percentage of academic sites [5], 
number of medications and arms [5], percentage of participants randomized to placebo [4], and drug company sponsorship of at least one study arm (medication donation alone was not considered company sponsorship [45]).

Drug-related factors. We classified the antipsychotics by their mechanisms according to the "Neuroscience-based Nomenclature" [46], antipsychotic doses in chlorpromazine equivalents according to the International-Consensus-Study-of AntipsychoticDosing [6, 47], and fixed vs. flexible dose studies [4].

We also analyzed whether the degree of placebo response in the studies was associated with the degree of drug response. We performed all analyses using Stata 14.2 and assuming a significance level of $5 \%$.

\section{RESULTS}

Description of included studies

The PRISMA flow diagram [8] is presented in the online supplement Fig. S1 and a description of the included studies in supplement Table S4. A summary of study characteristics is presented in Table 1. Overall, 167 studies with 28,102 participants met the inclusion criteria, of which 104 studies with 23,567 participants (8023 allocated to the placebo groups, and 15,544 to the drug groups), which were published between 1969 and 2016 provided data to calculate drug-placebo differences. In the studies with such data, the patients' mean duration of illness was 13.8 (SD 4.0) years, the mean age 38.6 (SD 4.8) years and the median duration of studies with useable outcomes was 6 weeks (range 3-26 weeks, for the outcome examined here all studies except one ( 26 weeks) lasted $\leq 12$ weeks). There were no studies in firstepisode patients or in treatment-resistant patients. Risk of bias is presented in the online supplement S5. We only included randomized, double-blind trials, but the reports often did not indicate full details about sequence generation, or allocation concealment. Descriptions of methods and success of blinding were frequently insufficient, as well. The data reflected the high dropout rates in current schizophrenia studies (overall mean $39.3 \%$, SD 17.1). Older studies were poorly reported, making it often impossible to extract data (52 studies (50\%) of the had a high risk of selective reporting). Sixty-five studies (62.5\%) were sponsored by the manufacturers of one antipsychotic included, 31 (29.8\%) were not primarily industry sponsored and in 8 (7.7\%) studies the sponsor was unclear.

Analysis of potential moderators-univariable analysis

The mean drug response in PANSS units was $17.45(95 \% \mathrm{Cl}$ $15.89,19.01 ; 100$ studies $(N)$ with 14,933 participants $(n))$, the placebo response was $6.25(95 \% \mathrm{Cl} 4.64,7.85 ; 99$ studies $(N)$ with 7623 participants $(n))$ and the mean SMD was $0.47(95 \% \mathrm{Cl}$ $0.42,0.52, p<0.001)$. As expected, drug-response and placeboresponse were strongly correlated (Fig. 1).

In the following text, in Table 2 and in Fig. 2 we sorted the moderators in the following way:

1. We first present the moderators that had a significant effect on the drug-placebo difference (effect size, SMD), because it is the drug-placebo difference that ultimately counts.

2. We then present the results of the moderators that had a significant effect on either drug-response or placeboresponse, but without an important effect on the resulting drug-placebo difference.

3. The moderators that had no important effect on any result are summarized at the end.

Moderators with a significant effect on drug-placebo differences (SMDs). Placebo-response was significantly higher in more recent studies, in studies with a larger number of participants and sites, in studies with a minimum baseline severity as an inclusion criterion, use of PANSS instead of the BPRS and in studies with operationalized diagnostic criteria, whereas drug-response was not significantly associated with these factors (Fig. 2a-l). The net effect were smaller drug-placebo differences (Fig. 2a, b, c, e, i, j and Table 1).

Studies using higher mean doses in chlorpromazine equivalents had significantly smaller placebo-response and tended to have more drug response (not significant), resulting in larger drugplacebo differences (Fig. 2d and Table 1).

The chronicity measures (mean age and mean duration of illness) were negatively correlated with both drug-response and placebo-response. However, the effect was more pronounced in the drug-groups, leading to smaller drug-placebo differences in more chronic patients, although this effect was significant only for mean age (Fig. $2 \mathrm{f}, \mathrm{g}$ ).

Industry sponsorship, the number of medications and drug mechanism according to the "Neuroscience-based Nomenclature $(\mathrm{NbN})$ " [46] had no significant impact on neither drug-response nor placebo-response, but the slopes were different enough that drug-placebo differences were significantly smaller in industrysponsored studies, in studies on drugs with another primary mechanism than $\mathrm{D}_{2}$-antagonism by $\mathrm{NbN}$ and in studies with only one medication (Fig. 2h, k, I and Table 1).

A post-hoc sensitivity analysis following a reviewer request showed that the pattern of increasing placebo response, relatively stable drug response and decreasing drug placebo differences was present before and after the year 2000 . The mean coefficients were virtually the same, although confidence intervals show that not all result that were statistically significant in the main analysis were significant in the sensitivity analysis. The smaller number of trials is a likely explanation (Supplement Fig. S2).

Moderators with a significant effect on drug-response and/or placebo-response but without a significant impact on drug-placebo differences (SMDs). Studies with higher baseline severity, shorter duration of the wash-out phases, shorter study duration, and studies conducted in countries outside the US or mixed had higher drug-response than their counterparts (Fig. 2m-p). However, placebo response was affected in the same direction with similar slopes resulting in no significant effect on drug-placebo differences (Fig. $2 m-p$ and Table 2).

Moderators without a significant effect on neither drug-response, nor placebo-response nor drug-placebo differences (SMDs). The total dropout rate, the percentage of patients randomized to placebo, the number of arms, the percentage of academic sites, fixed or flexible dosing, percentage of men, and risk of bias in terms of randomization, allocation concealment, blinding and missing outcome data had no important impact on the results (Fig. 2q-z).

Moderators of drug response, placebo response and drug-placebo differences - multivariable analysis

As some significant predictors are naturally related to each other, we made the following choices for the multivariable models:

a. We chose mean participant age rather than mean duration ill as a measure of chronicity, because more studies reported this outcome;

b. We did not include placebo response in the multivariable model of drug response and vice versa because both are strongly correlated (see Fig. 1).

Significant factors in the model for drug response included: average age and baseline severity; in the model of placebo response: average age and total number of participants; and for drug-placebo differences: degree of placebo response and 
Table 1. Summary of characteristics of included studies

\begin{tabular}{|c|c|c|c|c|c|c|}
\hline Explanatory variable & Mean & Standard deviation & Range & Median & $\mathrm{IQR}$ & Percentages \\
\hline Publication year & 2000 & 13.3 & $(1969,2016)$ & & & \\
\hline Total participants & 227 & 181.1 & $(12,729)$ & & & \\
\hline Number of sites & & & $(1112)$ & 25 & $(1,45)$ & \\
\hline Mean dose in chlorpromazine equivalents & 584.3 & 248.9 & $(200,1740)$ & & & \\
\hline Minimum severity entry criterion & & & & & & No: $28(26.9 \%) Y e s: ~ 73(70.2 \%)$ Unclear: 3(2.9\%) \\
\hline Mean age (years) & 38.6 & 4.8 & $(25.9,69.8)$ & & & \\
\hline Duration of illness (years) & 13.8 & 4.0 & $(2.5,32.0)$ & & & \\
\hline Industry sponsorship & & & & & & $\begin{array}{l}\text { Non-sponsored: } 31(29.8 \%) \text { Sponsored/Mixed: } 65(62.5 \%) \\
\text { Unclear: } 8(7.7 \%)\end{array}$ \\
\hline Scale & & & & & & PANSS: 33(31.7\%)BPRS: 68(65.4\%)Other: 3(2.9\%) \\
\hline Operationalized criteria & & & & & & Op: 88 (84.7\%)Not op:15 (14.4\%)Unlcear: 1(1.0\%) \\
\hline Number of medications & & & & & & 2 medications:33(31.7\%) > 2 medications:71 $(68.3 \%)$ \\
\hline Baseline severity (PANSS total) & 94.6 & 5.1 & $(80,112.5)$ & & & \\
\hline Duration of wash-out period (days) & & & $(0,84)$ & 3 & $(2,5)$ & \\
\hline Study duration (weeks) & & & $(3,26)$ & 6 & $(6,6)$ & \\
\hline Country & & & & & & USA: 45(43.3\%)Other: 59(56.7\%) \\
\hline Total dropout rate & $39.3 \%$ & $17.1 \%$ & $(0,82.6 \%)$ & & & \\
\hline Percentage randomized to placebo & $28.3 \%$ & $8.0 \%$ & $(14.1,50.4)$ & & & \\
\hline Number of arms & & & & & & 2 arms: $11(10.6 \%)>2$ arms: $93(89.4 \%)$ \\
\hline Percentage of academic sites & $57.6 \%$ & $36.3 \%$ & $(0100 \%)$ & & & \\
\hline Dosing schedule & & & & & & Fixed: 78(75\%)Flexible: $26(25 \%)$ \\
\hline Percentage of men & $66.3 \%$ & $20.6 \%$ & $(0100 \%)$ & & & \\
\hline Risk of bias: randomization & & & & & & Low risk: 48(46.2\%)Unclear: 56 (53.9\%) \\
\hline Risk of bias: allocation concealment & & & & & & Low risk: 33 (31.7\%)Unclear: 71(68.3\%) \\
\hline Risk of bias: blinding & & & & & & Low risk: 57 (54.8\%)Unclear: 47 (45.2\%) \\
\hline Risk of bias: missing outcome data & & & & & & Low risk: 73 (70.2\%)Unclear: 19 (18.3\%)High risk: 12(11.5\%) \\
\hline Drug Mechanism & & & & & & M1: 16 (15.4\%)Other mechanism: 88(84.6\%) \\
\hline
\end{tabular}

M1-M5 are drug mechanisms of action according to the "Neuroscience-based Nomenclature (NbN)" [46]: M1= receptor antagonists (D2) clopenthixol, fluphenazine, haloperidol, perphenzaine, pimozide, pipotiazine, sulpiride, trifluoperazine. M2 = receptor antagonists (D2, 5-HT2) chlorpromazine, iloperidone, loxapine, lurasidone, olanzapine, sertindole, thioridazine, ziprasidone, zotepine. M3 = receptor partial agonists (D2, 5-HT1A) aripiprazole, brexpiprazole, cariprazine. M4 = receptor antagonists (D2, 5- HT2, NE alpha2) asenapine, paliperidone, risperidone. M5 = receptor antagonist (D2, 5-HT2) and reuptake inhibitor (NET) quetiapine. A few old drugs have not been classified by $\mathrm{NbN}$ yet

IQR interquartile range, PANSS Positive and Negative Syndrome Scale, BPRS Brief Psychiatric Rating Scale, op. operationalized, non-op. not operationalized

industry sponsorship (industry-sponsored trials had smaller effect sizes than non-industry-sponsored ones, see Table 3).

\section{DISCUSSION}

In our analysis, we found that drug-response, placebo-response, and drug-placebo differences in effect sizes are not affected by the same factors in the same way. Certain factors influenced only either drug-response or on placebo-response so that they

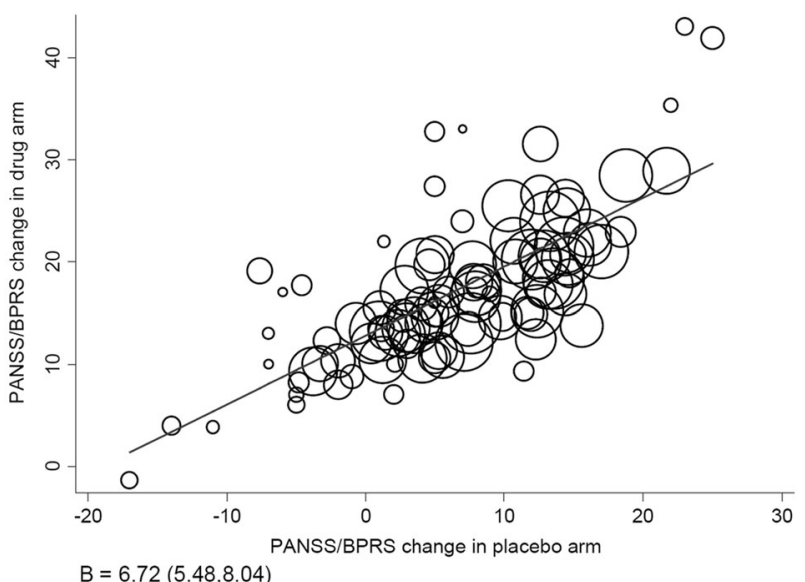

Fig. 1 Meta-regression placebo-response vs. drug-response had a net effect on drug-placebo differences. Other factors affected drug-response and placebo-response in the same direction and to a similar degree so that they had no net effect on the effect size. Finally, some factors had no influence for any of these relationships.

Factors that had an impact on drug-placebo differences (effect size)

We have previously demonstrated that placebo-response has increased over the years while drug-response remained stable resulting in decreasing drug-placebo differences (Fig. 2a [1]). Here, we found a similar pattern of results for factors "sample size" and the related factor "number of sites" (Fig. 2b, c). Specifically, the more participants and sites, the more placebo-response and the smaller the effect sizes, while drug-response was unaffected by sample size and number of sites. The reason for this is difficult to interpret. We speculate that drug-response may plateau, while in studies with large sample size more patients who benefit from placebo are recruited compared to smaller trials where patients can be more carefully selected.

The effects on drug response and placebo response differed even more with regard to the doses used. In studies with high antipsychotic doses drug-response increased while placeboresponse decreased and consequently effect size increased (Fig. 2d). It could be that the patients in studies with high doses are more severely ill so that they benefit more from drug and less from placebo. An alternative interpretation is that in studies with high doses there is more un-blinding by side-effects so that raters may guess the group patients are assigned to. 


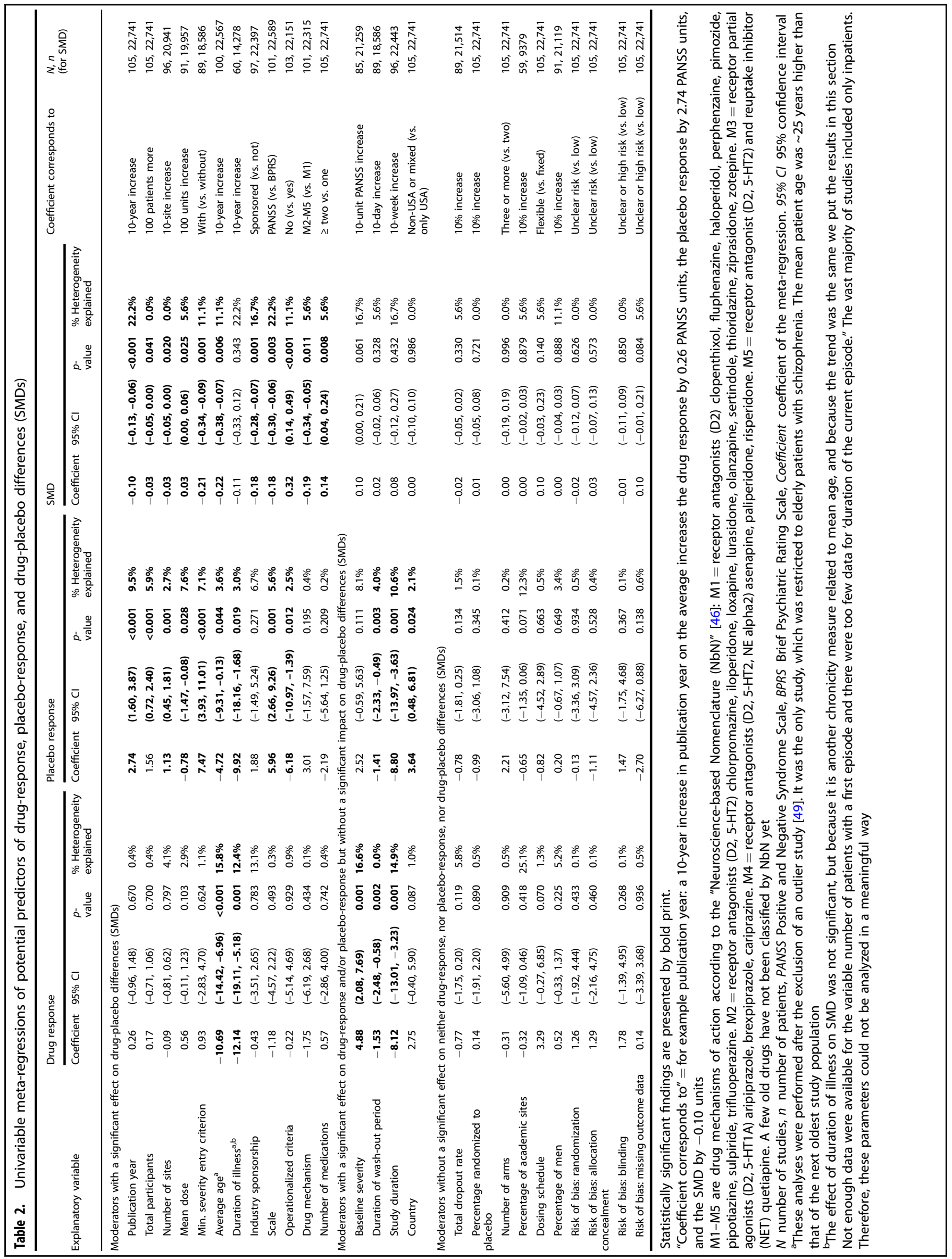


a) - I) Moderators with a significant effect on drug-placebo differences (SMDs)
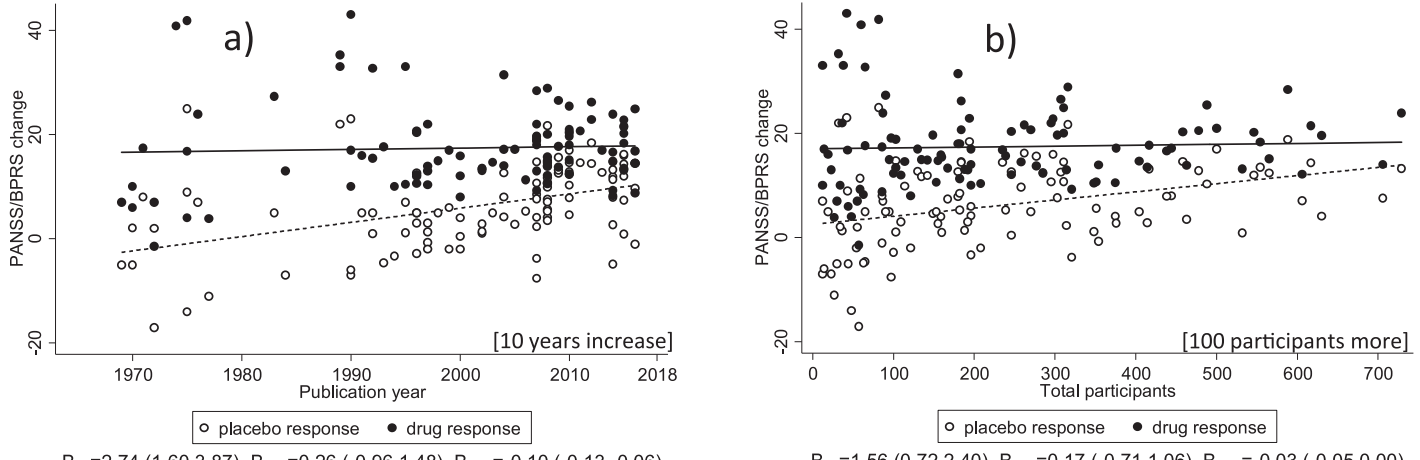

$B_{p 1}=2.74(1.60,3.87), B_{d n g}=0.26(-0.96,1.48), B_{S M D}=-0.10(-0.13,-0.06)$

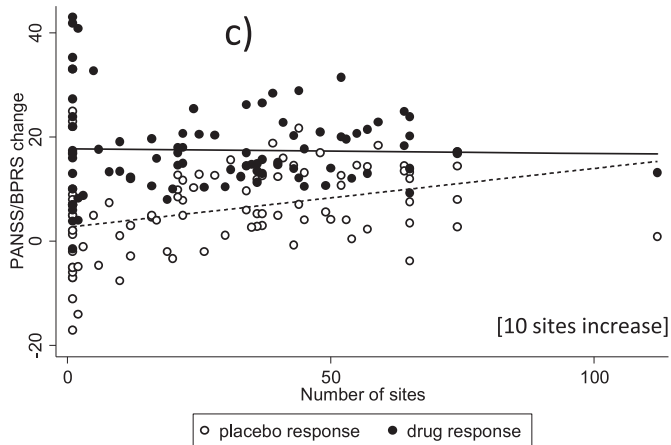

$B_{\text {pla }}=1.13(0.45,1.81), B_{\text {drug }}=-0.09(-0.81,0.62), B_{\text {SMD }}=-0.03(-0.05,0.00)$
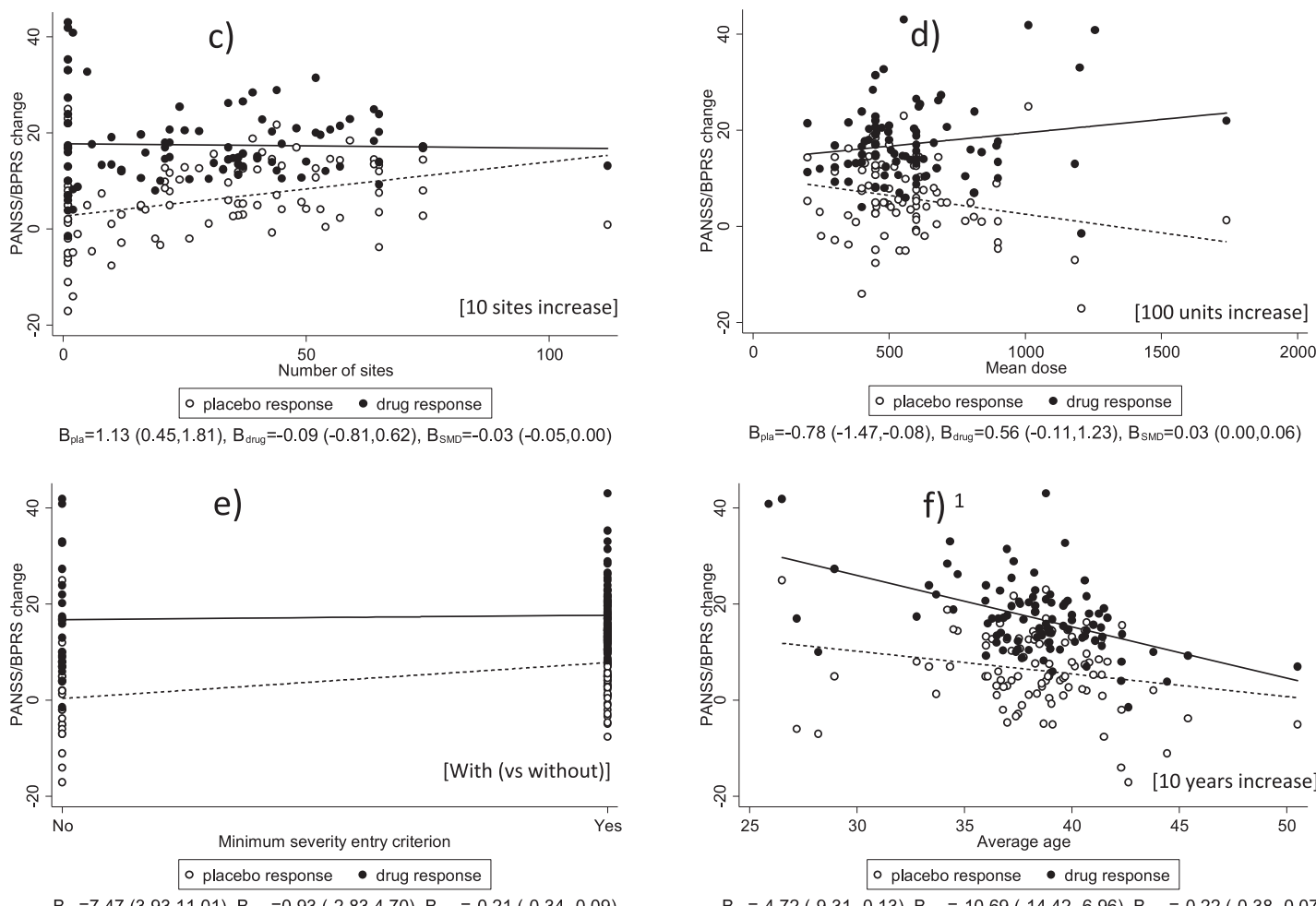

$B_{p l a}=-0.78(-1.47,-0.08), B_{d n g}=0.56(-0.11,1.23), B_{\text {sMD }}=0.03(0.00,0.06)$

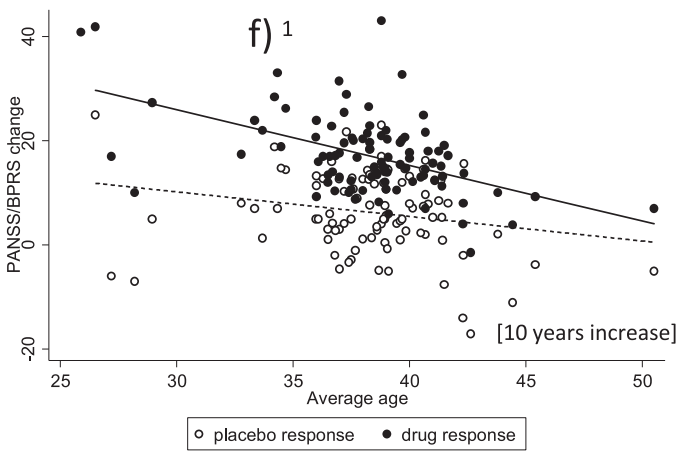

$B_{\mathrm{pla}}=-4.72(-9.31,-0.13), B_{\text {drug }}=-10.69(-14.42,-6.96), B_{\text {sMD }}=-0.22(-0.38,-0.07)$

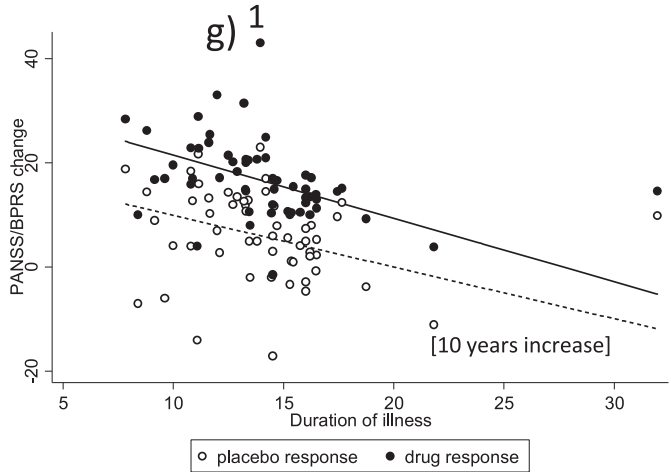

$B_{\text {pla }}=-9.92(-18.16,-1.68), B_{d n g}=-12.14(-19.11,-5.18), B_{\text {SMD }}=-0.11(-0.33,0.12)$

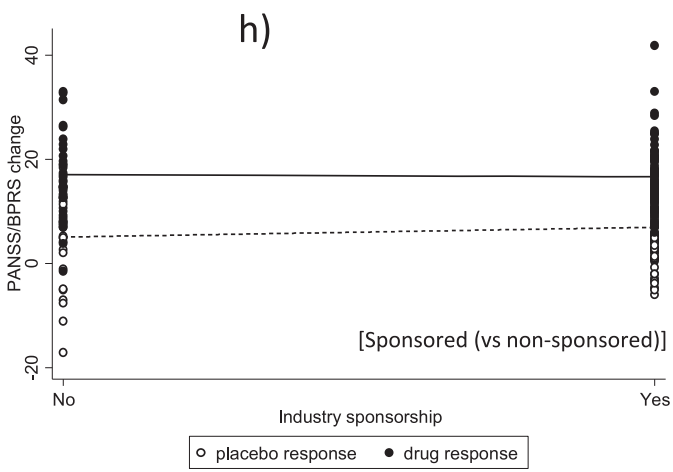

$B_{p l a}=1.88(-1.49,5.24), B_{d r a g}=-0.43(-3.51,2.65), B_{s M D}=-0.18(-0.28,-0.07)$ 

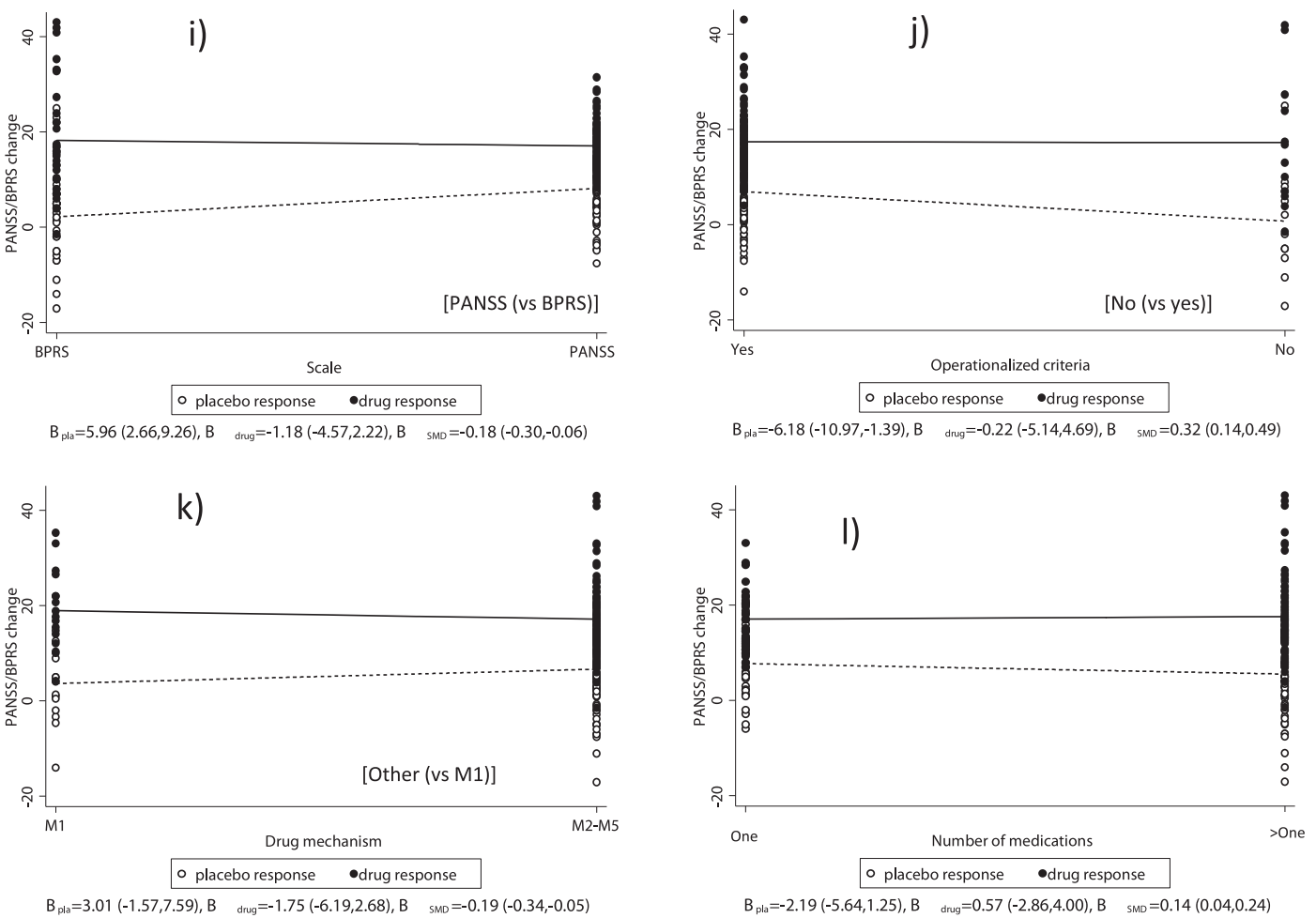

$\underline{2 m})-p)$ Moderators with a significant effect on drug-response and/or placebo-response but without a significant impact on drug-placebo differences (SMDs)
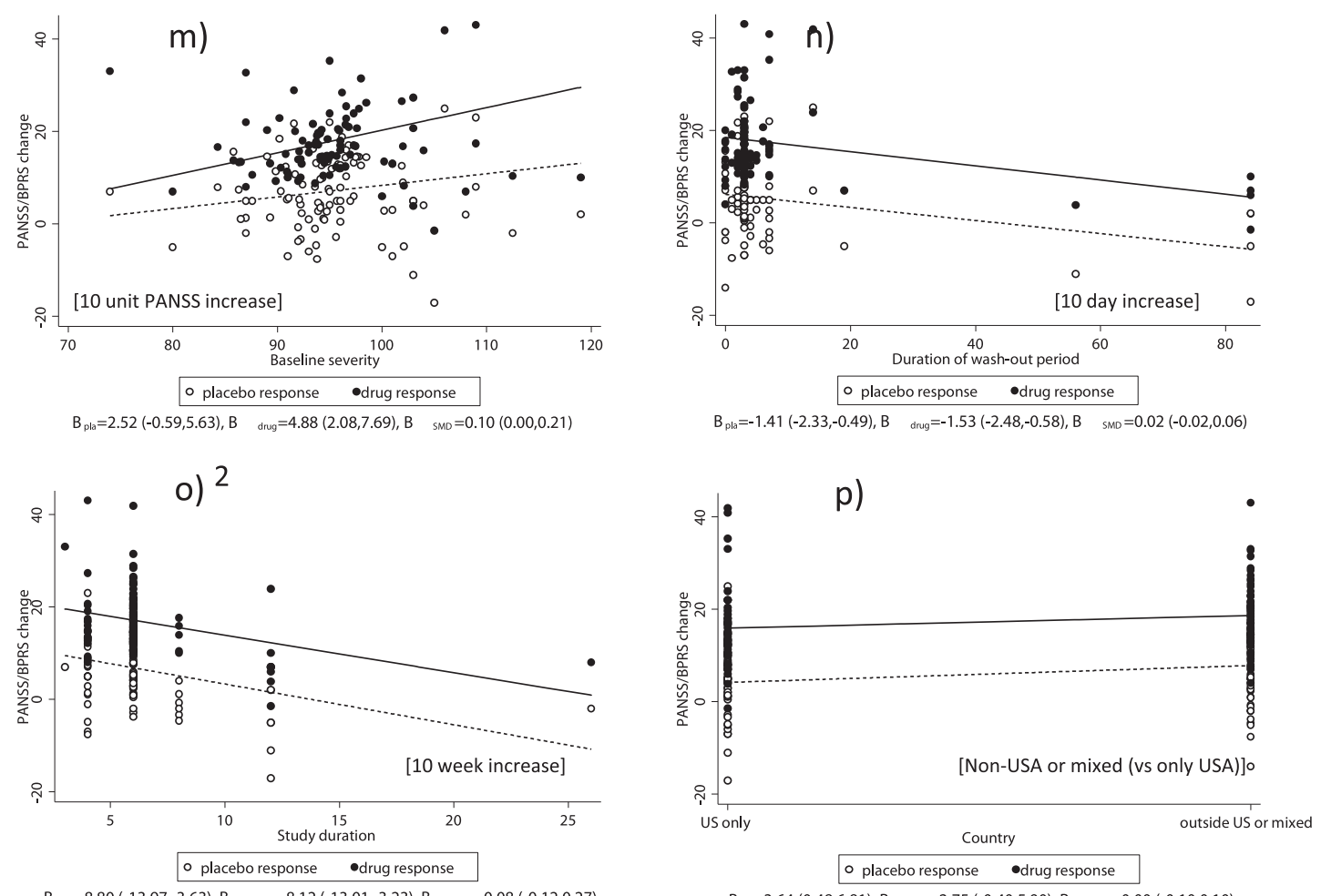

$B_{\text {pla }}=-8.80(-13.97,-3.63), B \quad$ drug $=-8.12(-13.01,-3.23), B \quad$ smo $=0.08(-0.12,0.27)$ $B_{\text {pla }}=3.64(0.48,6.81), B \quad$ drug $=2.75(-0.40,5.90), B \quad$ SMD $=0.00(-0.10,0.10)$ 
2a) - z) Moderators without a significant effect on neither drug-response, nor placebo-response, nor drug-placebo differences (SMDs)
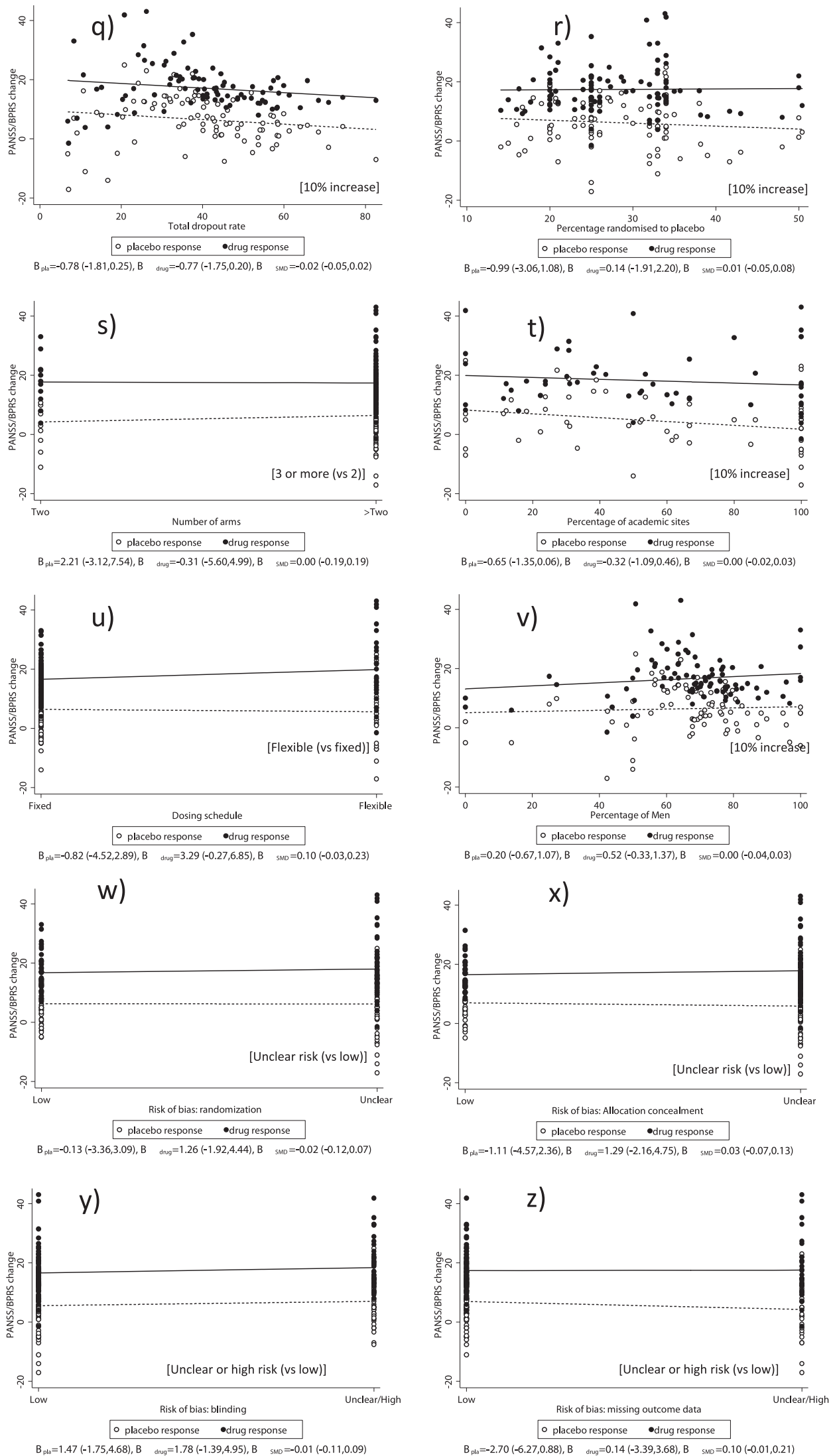
Fig. 2 Moderators of placebo-response-univariable meta-regressions. The figures in this panel correspond to the following moderators: a Publication year, $\mathbf{b}$ number of participants, $\mathbf{c}$ )number of sites, $\mathbf{d}$ mean dose in chlorpromazine equivalents, e minimum scale-derived severity threshold as inclusion criterion, $\mathbf{f}$ average participant age in years, $\mathbf{g}$ average duration of illness in years, $\mathbf{h}$ industry sponsorship, $\mathbf{i}$ scale, $\mathbf{j}$ operationalized criteria or not, $\mathbf{k}$ drug mechanism, I number of medications, $\mathbf{m}$ baseline severity (PANSS total score at baseline) $\mathbf{n}$ duration of wash-out phases, $\mathbf{o}$ study duration, $\mathbf{p}$ country, $\mathbf{q}$ total dropout rate, $\mathbf{r}$ percentage randomized to placebo, $\mathbf{s}$ number of arms risk, $\mathbf{t}$ percentage of academic sites, $\mathbf{u}$ dosing schedule, $\mathbf{v}$ percentage of men, $\mathbf{w}$ risk of bias concerning randomization method, $\mathbf{x}$ risk of bias concerning allocation concealment, $\mathbf{y}$ risk of bias concerning blinding, $\mathbf{z}$ risk of bias concerning missing outcomes. M1-M5 are drug mechanisms of action according to the "Neuroscience-based Nomenclature (NbN)" [46]: M1 = receptor antagonists (D2) clopenthixol, fluphenazine, haloperidol, perphenzaine, pimozide, pipotiazine, sulpiride, trifluoperazine. M2 = receptor antagonists (D2, 5-HT2) chlorpromazine, iloperidone, loxapine, lurasidone, olanzapine, sertindole, thioridazine, ziprasidone, zotepine. M3 = receptor partial agonists (D2, 5-HT1A) aripiprazole, brexpiprazole, cariprazine. $\mathrm{M} 4=$ receptor antagonists (D2, 5-HT2, NE alpha2) asenapine, paliperidone, risperidone. M5 = receptor antagonist (D2, 5-HT2) and reuptake inhibitor (NET) quetiapine. A few old drugs have not been classified by $\mathrm{NbN}$ yet. The $B$-values and their $95 \%$ confidence intervals at the bottom of the graphs are the coefficients for placebo response $\left(B_{\mathrm{pla}}\right)$, drug response $\left(B_{\mathrm{drug}}\right)$, and drug-placebo differences $\left(B_{\mathrm{SMD}}\right)$. The numbers in square brackets in the figures describe to what the coefficient refers to. For example, in a publication year: " [10 years increase], $B_{\text {pla }}=2.74(1.60,3.88), B_{\text {drug }} 0.26(-0.96,1.48), B_{S M D}\left(-0.10(-0.13,-0.06)^{\prime \prime}\right.$ means a study that was conducted 10 years later had on average 2.74 (95\% confidence interval 1.60 to 3.88) PANSS points higher placebo response, $0.26(-0.96,1.48)$ higher drug response and $-0.10(-0.13,-0.06)$ lower drug-placebo difference. Or in $\mathbf{g}$, as an example for a dichotomous moderator: [With (vs. not)], $B_{\text {pla }}=7.47\left(3.93,11.01\right.$ ), $B_{\text {drug }}=0.93$ $(-2.83,4.70), B_{S M D}=-0.21(-0.34,-0.09) "$ means a study, which had a minimum baseline severity as an inclusion criterion had on average 7.47 (95\% confidence interval $3.93,11.01)$ PANSS points higher placebo response, $-0.21(-0.34,-0.09)$ lower drug response and $-0.21(-0.34,-0.09)$ lower drug-placebo difference compared to a study without such a criterion. The moderators are statistically significant if the $95 \%$ confidence interval does not include $1 .{ }^{1}$ Results without one outlier, which was the only study restricted to elderly people with schizophrenia who had a mean age of 70 years, 20 years more than the next oldest study population, ${ }^{2}$ this meta-regression was also statistically significant when the only outlier study of a duration of 26 weeks was excluded

In studies that employed a threshold for minimum severity as an inclusion criterion we found that placebo-response was higher than in studies without such a criterion, while drug-response is not affected (Fig. 2e). One reason could be artificial baseline inflation in such studies. If the baseline ratings were inflated to meet an inclusion criterion, the next rating of patients in the placebo-group may be automatically lower, while in the drug group we may see a true reduction of symptoms. More chronic patients (the moderator mean age) respond less well to drug and to placebo but the effect was more pronounced in the drug groups so that it could be helpful to recruit younger patients than it is currently the case (mean age around 40 years, Fig. 2f). If, as in some previous reports $[3$, 4], we would have only analyzed placebo-response, the conclusion might have been to use more chronic patients to avoid placebo-response, demonstrating the importance of examining drug-response simultaneously. For the related factor "duration of illness" the influence on the drug-placebo difference was not significant, but fewer studies reported this factor and the pattern was the same (Fig. $2 \mathrm{~g}$ ).

Industry-sponsoring tended to reduce drug response and tended to increase placebo response, which resulted in smaller effect sizes than those of non-sponsored studies (Fig. 2h). This finding is counterintuitive in the sense that the pharmaceutical industry is suspected for designing trials in a way that inflates positive results [45]. However, industry sponsorship probably is a composite of multiple factors.

Studies using PANSS rather than BPRS, studies analyzing other antipsychotics than $\mathrm{D}_{2}$ receptor antagonists according to $\mathrm{NbN}$, and studies applying operationalized diagnostic criteria had higher placebo-response, similar drug-response and smaller drug-placebo differences than their counterparts (Fig. 2i-k). However, this may be confounded by publication year. In early studies operationalized diagnostic criteria and the PANSS were not available yet, and early studies examined $D_{2}$ receptor antagonists (mainly haloperidol) more frequently than recent studies.

Finally, studies that examined two or more active drugs had larger drug-placebo differences than those with only one drug. This result stands in contrast the finding that the number of arms in a study (which could also be due to different dose arms of the same drug being examined) did not affect the effect size (Fig. 2l, s).

Factors that had an impact on either drug-response or placeboresponse or both, but not on drug-placebo differences

Baseline severity data shows a pattern that could be expected based on the findings from a previous individual-patient data meta-analysis [36]. The more ill the patients were at baseline the higher the difference between drug-response and placeboresponse, although the effect on the drug-placebo difference was not significant (Fig. $2 \mathrm{~m}$ ). In contrast, the factors: duration of the wash-out period, study duration and study origin affected drug-response and placebo-response in the same way so that they had no impact on the drug-placebo difference (Fig. $2 n-p)$. This finding is important, because from previous analyses examining placebo-response only, one might have concluded that washout phases need to be long enough to have a low placebo-response. However, our data suggest that longer wash-out phases may not be an optimal solution, because drug-response was also lower in studies with long wash-out periods.

Finally, the factors total dropout rate, percentage randomized to placebo, number of arms, percentage of academic sites, fixed or flexible dosing, and the various risk of bias items had no important effect on drug-response, placebo-response and drug-placebo differences (Fig. 2q-z). Reasons why, in contrast to some of our previous analyses, we found an effect of the percentage of trial participants randomized to placebo group [4, 5], the percentage of academic sites [5] or the percentage of men in a study [37], could be due to somewhat different definitions, or it could simply be due to our much larger number of trials available for the current analysis.

Given that various factors did not affect drug-response, placebo-response and drug-placebo differences in the same way, it is not surprising that the factors which were significant in the multivariable analyses were also not the same (Table 3). It should be noted that multivariable models are subject to multicollinearity and sometimes lead to over-simplified models by erroneously dropping variables with important contribution to model fit. In contrast, univariable models have a risk of confounding as moderators are correlated. Both results should therefore be considered. To our knowledge the publication by Rutherford et al. $[6,47]$ is the only other one that analyzed the interaction between drug-response and placebo-response. Comparison is difficult, because at that time only approximately half of the placebo-controlled studies were available, and because studies comparing antipsychotics with each other were included as well. This is probably the reason why drug-response increased in their analysis while it remained stable in ours.

Our analysis is limited by the fact that we have examined antipsychotics as a class, because not enough studies would have been available for single drugs. Moreover, meta-regression is based on mean values of trials, which makes the results prone to ecological bias [48], meaning that an average value representing a 


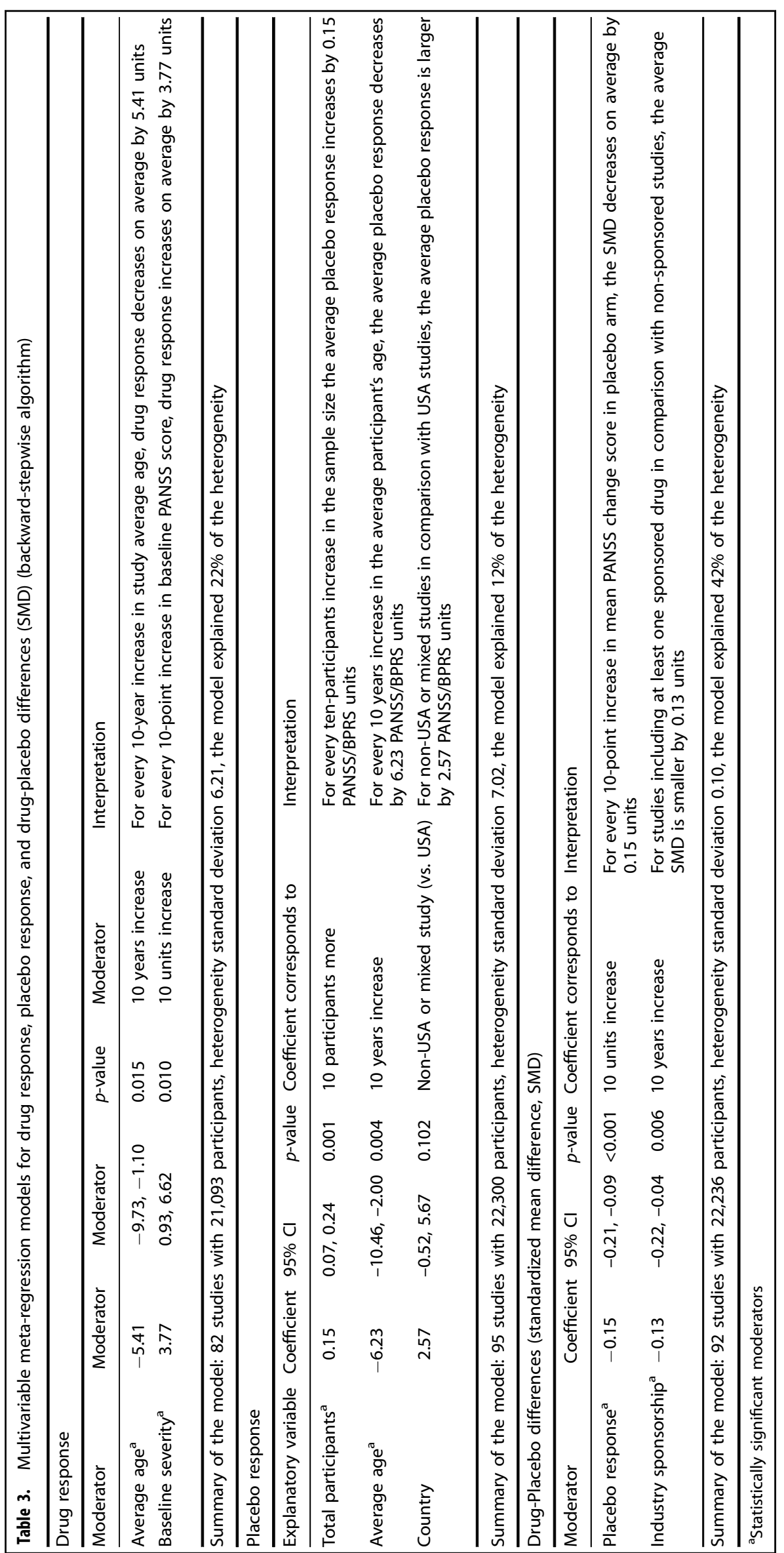


large group of individuals is rarely accurate in describing a specific individual from that group. Individual patient data meta-analysis is more powerful in this regard, but unfortunately it is limited by insufficient data availability.

Previous analyses had shown that placebo-response in antipsychotic drug trials has increased over the years $[3,5,6]$, that drug response has remained stable [1] and that as a consequence drug-placebo differences have decreased [1]. The current analysis adds the following information to these analyses: The predictors of drug response are not the same as those of placebo response and of effect sizes. Therefore, it is not sufficient to only understand placebo response in such trials. We also need to understand drugresponse, because its interaction with placebo-response forms the drug-placebo difference, which counts for patients.

\section{FUNDING AND DISCLOSURE}

The meta-analysis was supported by the German Federal Ministry for Education and Research (Bundesministerium für Bildung und Forschung, BMBF) Grant: FKZ 01KG1115 and by the National Institute for Health Research (NIHR) Oxford Health Biomedical Research Center (grant BRC-1215-20005). The funding body was not involved in the study design, collection, analysis, interpretation of data, and in the decision to submit for publication. The views expressed are those of the authors and not necessarily those of the UK National Health Service, the NIHR, or the UK Department of Health. Authors had full access to the study data and complete discretion in the analysis of data and writing of this report. In the last 3 years, Stefan Leucht has received honoraria for consulting or lectures from LB Pharma, Otsuka, Lundbeck, Boehringer Ingelheim, LTS Lohmann, Janssen, Johnson\&Johnson, TEVA, MSD, Sandoz, SanofiAventis, Angelini, Recordati, Sunovion, Geodon Richter. Claudia Leucht is Stefan Leucht's spouse. Maximilian Huhn received lecture honoraria from Janssen and Lundbeck. Dr. Cipriani is also supported by an NIHR Research Professorship (grant RP-2017-08-ST2-006) and by the NIHR Oxford Cognitive Health Clinical Research Facility. The other authors declare no competing interests.

\section{ACKNOWLEDGEMENTS}

We thank Georgia Salanti for her initial help in developing the statistical method Samantha Roberts for her help in the literature search, Magdolna Tardy, M.Sc, for her help in study selection and Marc Krause, Philipp Rothe, MD, Thomas Arndt and Matteo Rabaioli, Susanne Bächer, Natalie Peter for help in data extraction. Ole Andreassen, Guy Chouinard, Michael Jann, Herbert Meltzer, Ofer Agid, Bret Rutherford, Merck, Dainippon Sumitomo, Novartis, Sunovion, EliLilly, Johnson\&Johnson and Pfizer have replied to our requests for explanations or data. We could also again use the data that authors had sent us for requests in the context of previous meta-analyses of our group.

\section{AUTHOR CONTRIBUTIONS}

$\mathrm{SL}, \mathrm{A}$ Chaimani, DM, and JMD were involved in designing the review. $\mathrm{MH}, \mathrm{MT}$, and $\mathrm{SL}$ identified and acquired reports of trials. $\mathrm{CL}, \mathrm{MH}, \mathrm{BH}, \mathrm{MS}, \mathrm{MR}, \mathrm{SB}, \mathrm{MK}, \mathrm{PR}, \mathrm{TA}, \mathrm{NP}$, and $\mathrm{SL}$ extracted data. $\mathrm{SL}$ and $\mathrm{CL}$ contacted authors of trials and pharmaceutical industries for additional information. SL, A Chaimani, and DM analyzed and interpreted the data. A Chaimani and JRG contributed to the interpretation of the data. SL, A Chaimani, A Cipriani, and BH drafted the manuscript and all other authors critically reviewed the manuscript for important intellectual content. All authors approved the final version.

\section{ADDITIONAL INFORMATION}

Supplementary Information accompanies this paper at (https://doi.org/10.1038/ s41386-019-0440-6)

Publisher's note: Springer Nature remains neutral with regard to jurisdictional claims in published maps and institutional affiliations.

\section{REFERENCES}

1. Leucht S, Leucht C, Huhn M, Chaimani A, Mavridis D, Helfer B, et al. Sixty years of placebo-controlled antipsychotic drug trials in acute schizophrenia: systematic review, bayesian meta-analysis, and meta-regression of efficacy predictors. Am J Psychiatry. 2017;174:appiajp201716121358.

2. Study 049. A 6-week, double-blind, randomized, fixed dose, parallel-group study of the efficacy and safety of three dose levels of SM-13496 (lurasidone) compared to placebo and haloperidol in patients with schizophrenia who are experiencing an acute exacerbation of symptoms. Center for drug evaluation and research. Application number 200603 Medical review(s) http://www.fgagov. 2010

3. Leucht S, Chaimani A, Leucht C, Huhn M, Mavridis D, Helfer B, et al. 60years of placebo-controlled antipsychotic drug trials in acute schizophrenia: Metaregression of predictors of placebo response. Schizophr Res. 2018;201:315-23.

4. Mallinckrodt $\mathrm{CH}$, Zhang L, Prucka WR, Millen BA. Signal detection and placebo response in schizophrenia: parallels with depression. Psychopharmacol Bull. 2010;43:53-74.

5. Agid O, Siu CO, Potkin SG, Kapur S, Watsky E, Vanderburg D, et al. Metaregression analysis of placebo response in antipsychotic trials, 1970-2010. Am J Psychiatry. 2013;170:1335-44.

6. Rutherford BR, Pott E, Tandler JM, Wall MM, Roose SP, Lieberman JA. Placebo response in antipsychotic clinical trials: a meta-analysis. JAMA psychiatry. 2014;71:1409-21.

7. Kirsch I. Are drug and placebo effects in depression additive? Biol Psychiatry. 2000;47:733-5.

8. Liberati A, Altman DG, Tetzlaff J, Mulrow C, Gotzsche PC, loannidis JP, et al. The PRISMA statement for reporting systematic reviews and meta-analyses of studies that evaluate health care interventions: explanation and elaboration. PLoS Med. 2009;6:e1000100.

9. Carpenter WT, Buchanan RW. Schizophrenia. N Eng J Med. 1994;330:681-90.

10. Leucht S, Tardy M, Komossa K, Heres S, Kissling W, Salanti G, et al. Antipsychotic drugs versus placebo for relapse prevention in schizophrenia: a systematic review and meta-analysis. Lancet. 2012;379:2063-71.

11. Leucht S, Cipriani A, Spineli L, Mavridis D, Orey D, Richter F, et al. Comparative efficacy and tolerability of 15 antipsychotic drugs in schizophrenia: a multipletreatments meta-analysis. Lancet. 2013;382:951-62.

12. Honigfeld G. Clozapine: antipsychotic activity in treatment-resistant schizophrenics. Adv Ther. 1984;1:77-97.

13. Samara MT, Cao H, Helfer B, Davis JM, Leucht S. Chlorpromazine versus every other antipsychotic for schizophrenia: a systematic review and meta-analysis challenging the dogma of equal efficacy of antipsychotic drugs. Eur Neuropsychopharmacol: J Eur Coll Neuropsychopharmacol. 2014;24:1046-55.

14. Hartling L, Abou-Setta AM, Dursun S, Mousavi SS, Pasichnyk D, Newton AS. Antipsychotics in adults with schizophrenia: comparative effectiveness of firstgeneration versus second-generation medications: a systematic review and meta-analysis. Ann Intern Med. 2012;157:498-511.

15. Dold M, Samara MT, Li C, Tardy M, Leucht S. Haloperidol versus first-generation antipsychotics for the treatment of schizophrenia and other psychotic disorders. Cochrane Database Syst Rev. 2015;1:CD009831.

16. McMahon RP, Kelly DL, Boggs DL, Li L, Hu Q, Davis JM, et al. Feasibility of reducing the duration of placebo-controlled trials in schizophrenia research. Schizophr Bull. 2008;34:292-301.

17. Higgins JPT, Green S. Cochrane handbook for systematic reviews of interventions version 5.1.0 [updated March 2011]. Chichester, UK: Wiley and Sons; 2011.

18. Wu TX, Li YP, Liu GJ, Bian Z, Li J, Zhang J, et al. Investigation of authenticity of 'claimed' randomized controlled trials (RCTs) and quality assessment of RCT reports published in China. Presented at the XIV Cochrane Colloquium, Dublin, Ireland, October 23-26; 2006.

19. Woodhead M. $80 \%$ of China's clinical trial data are fraudulent, investigation finds. BMJ. 2016;355:i5396.

20. Adams CE, Coutinho E, Davis JM, Duggan L, Essali $A$, Fenton $M$, et al. The cochrane library. Chichester, UK: John Wiley \& Sons Ltd; 2011.

21. Klein DF, Davis JM. Diagnosis and drug treatment of psychiatric disorders. Baltimore: Williams and Wilkins; 1969.

22. Adams CE, Awad G, Rathbone J. Chlorpromazine versus placebo for schizophrenia. Cochrane Database Syst Rev. 2007;Art. No.: CD000284.

23. Joy $C B$, Adams CE, Laurie S. Haloperidol versus placebo for schizophrenia. Cochrane Database Syst Rev. 2007;Art. No.: CD003082.

24. Shen X, Xia J, Adams CE. Flupenthixol versus placebo for schizophrenia. Cochrane Database Syst Rev. 2012;11:CD009777.

25. Matar $\mathrm{HE}$, Almerie MQ. Oral fluphenazine versus placebo for schizophrenia. Cochrane Database Syst Rev. 2007;24:CD006352.

26. Hartung B, Wada M, Laux G, Leucht S. Perphenazine for schizophrenia. Cochrane Database Syst Rev. 2005:CD003443.

27. Omori IM, Wang J. Sulpiride versus placebo for schizophrenia. Cochrane Database Syst Rev. 2009:CD007811. 
28. Fenton M, Rathbone J, Reilly J, Sultana A. Thioridazine for schizophrenia. Cochrane Database Syst Rev. 2007;6:CD001944.

29. Leucht S, Arbter D, Engel RR, Kissling W, Davis JM. How effective are secondgeneration antipsychotic drugs? A meta-analysis of placebo-controlled trials. MolPsychiatry. 2009;14:429-47.

30. Kay SR, Fiszbein A. The positive and negative symptome scale (PANSS) for schizophrenia. Schizophr Bull. 1987;13:261-75.

31. Overall JE, Gorham DR. The brief psychiatric rating scale. Psychol Rep. 1962;10:790-812.

32. Leucht $S$, Rothe $P$, Davis JM, Engel RR. Equipercentile linking of the BPRS and the PANSS. Eur Neuropsychopharmacol: J Eur Coll Neuropsychopharmacol. 2013;23:956-9.

33. Leon AC, Mallinckrodt CH, Chuang-Stein C, Archibald DG, Archer GE, Chartier K. Attrition in randomized controlled clinical trials: methodological issues in psychopharmacology. Biol Psychiatry. 2006;59:1001-5.

34. Furukawa TA, Barbui C, Cipriani A, Brambilla P, Watanabe N. Imputing missing standard deviations in meta-analyses can provide accurate results. J Clin Epidemiol. 2006:59:7-10.

35. Salanti G, Higgins JP, White IR. Bayesian synthesis of epidemiological evidence with different combinations of exposure groups: application to a gene-geneenvironment interaction. Stat Med. 2006;25:4147-63.

36. Furukawa TA, Levine SZ, Tanaka S, Goldberg Y, Samara M, Davis JM, et al. Initial severity of schizophrenia and efficacy of antipsychotics: participant-level metaanalysis of 6 placebo-controlled studies. JAMA Psychiatry. 2015;72:14-21.

37. Rabinowitz J, Werbeloff N, Caers I, Mandel FS, Stauffer V, Menard F, et al. Determinants of antipsychotic response in schizophrenia: implications for practice and future clinical trials. J Clin Psychiatry. 2014;75:e308-16.

38. Mattila T, Wohlfarth T, Koeter M, Storosum J, van den Brink W, de Haan L, et al. Geographic variation in efficacy of atypical antipsychotics for the acute treatment of schizophrenia-an individual patient data meta-analysis. Eur Neuropsychopharmacol: J Eur Coll Neuropsychopharmacol. 2014;24:1067-77.

39. Rabinowitz J, Levine SZ, Barkai O, Davidov O. Dropout rates in randomized clinical trials of antipsychotics: a meta-analysis comparing first- and second-generation drugs and an examination of the role of trial design features. Schizophr Bull. 2009;35:775-88.

40. Levine SZ, Goldberg Y, Samara M, Davis JM, Leucht S. Joint modeling of dropout and outcome in three pivotal clinical trials of schizophrenia. Schizophr Res. 2015;164:122-6.

41. Schulz KF, Chalmers I, Hayes RJ, Altman DG. Empirical evidence of bias: dimensions of methodological quality associated with estimates of treatment effects in controlled trials. JAMA. 1995;273:408-12.

42. Wood L, Egger M, Gluud LL, Schulz KF, Juni P, Altman DG, et al. Empirical evidence of bias in treatment effect estimates in controlled trials with different interventions and outcomes: meta-epidemiological study. BMJ. 2008;336:601-5.

43. Porta N, Bonet C, Cobo E. Discordance between reported intention-to-treat and per protocol analyses. J Clin Epidemiol. 2007;60:663-9.

44. Egger M, Davey mG, Schneider M, Minder C. Bias in meta-analysis detected by a simple, graphical test. BMJ. 1997;315:629-34.

45. Heres S, Davis J, Maino K, Jetzinger E, Kissling W, Leucht S. Why olanzapine beats risperidone, risperidone beats quetiapine, and quetiapine beats olanzapine: an exploratory analysis of head-to-head comparison studies of second-generation antipsychotics. Am J Psychiatry. 2006;163:185-94.

46. Zohar J, Stahl S, Moller HJ, Blier P, Kupfer D, Yamawaki S, et al. A review of the current nomenclature for psychotropic agents and an introduction to the Neuroscience-based Nomenclature. Eur Neuropsychopharmacol. 2015; 25:2318-25.

47. Gardner DM, Murphy AL, O'Donnell H, Centorrino F, Baldessarini RJ. International consensus study of antipsychotic dosing. Am J Psychiatry. 2010;167:686-93.

48. Simmonds MC, Higgin JP. Covariate heterogeneity in meta-analysis: criteria for deciding between meta-regression and individual patient data. Stat Med. 2007;26:2982-99.

49. Tzimos A, Samokhvalov V, Kramer M, Ford L, Gassmann-Mayer C, Lim P, et al. Safety and Tolerability of Oral Paliperidone Extended-Release Tablets in Elderly Patients With Schizophrenia: A Double-Blind, Placebo-Controlled Study With Six-Month Open-Label Extension Am J Geriatr Psychiatry. 2008;16:31-43. 